\title{
Multiplicity and uniqueness results for the singular nonlocal boundary value problem involving nonlinear integral conditions
}

Baoqiang Yan ${ }^{1}$, Donal O'Regan ${ }^{2,3}$ and Ravi P Agarwal ${ }^{3,4^{*}}$

\footnotetext{
"Correspondence: agarwal@tamuk.edu ${ }^{3}$ Department of Mathematics, Nonlinear Analysis and Applied Mathematics (NAAM), King Abdulaziz University, Jeddah, Saudi Arabia

${ }^{4}$ Department of Mathematics, Texas A\&M University-Kingsville, Kingsville, TX 78363, USA

Full list of author information is available at the end of the article
}

\section{Springer}

\begin{abstract}
In this paper, using fixed point index and the mixed monotone technique, we present some multiplicity and uniqueness results for the singular nonlocal boundary value problems involving nonlinear integral conditions. Our nonlinearity may be singular in its dependent variable and it is allowed to change sign.
\end{abstract}

\section{Introduction}

In this paper, we consider the existence of positive solutions of nonlinear nonlocal boundary value problem (BVP) of the form

$$
-y^{\prime \prime}=q(t) f(t, y(t)), \quad t \in(0,1)
$$

with integral boundary conditions

$$
y(0)=\alpha[y]=\int_{0}^{1}(y(s))^{a} d A(s), \quad y(1)=\beta[y]=\int_{0}^{1}(y(s))^{b} d B(s)
$$

involving Stieltjes integrals, $a \geq 0, b \geq 0$.

In [1], using the Leray-Schauder alternative, Z. Yang considered the problem

$$
-y^{\prime \prime}=f(y(t)), \quad t \in(0,1)
$$

with integral boundary conditions

$$
y(0)=\alpha[y]=\int_{0}^{1} y(s) d A(s), \quad y(1)=\beta[y]=\int_{0}^{1} y(s) d B(s)
$$

and discussed the existence and uniqueness of a positive solution for BVP (1.3)-(1.4) with a sign-changing nonlinearity $f$. C.S. Goodrich discussed (1.1) with nonlinear integral conditions

$$
y(0)=H_{1}\left(\int_{0}^{1} y(s) d A(s)\right)+\int_{E} H_{2}(s, y(s)) d s, \quad y(1)=0,
$$

( 2014 Yan et al.; licensee Springer. This is an open access article distributed under the terms of the Creative Commons Attribution License (http://creativecommons.org/licenses/by/2.0), which permits unrestricted use, distribution, and reproduction in any medium, provided the original work is properly cited. 
where $E \subseteq(0,1)$ is some measurable set (see [2]). Moreover, there are some interesting results when the measures are signed (see [3-5]). Using the mixed monotone technique, L. Kong considered

$$
-y^{\prime \prime}=\lambda f(t, y(t)), \quad t \in(0,1)
$$

with (1.4) and discussed the uniqueness of positive solutions (see [4]). J.R.L. Webb discussed the multiplicity of positive solutions for BVP (1.5)-(1.4) when $f(t, y)$ is positive and continuous on $(0,1) \times[0,+\infty)$; note that $f$ has no singularities at $y=0$ (see [5]). Using the fixed point index, G. Infante discussed (1.1) with nonlinear integral boundary conditions (see [3]),

$$
y(0)+H_{1}\left(\int_{0}^{1} y(s) d A(s)\right)=0, \quad y(1)+u(\eta)=H_{2}\left(\int_{0}^{1} y(s) d B(s)\right) .
$$

Inspired by the above works and [6-16], we consider the BVP (1.1)-(1.2) when $f$ is singular at $y=0$ and $f$ may be sign changing. Using the fixed point index and the mixed monotone technique we establish some new existence results for the BVP (1.1)-(1.2).

Our paper is organized as follows. In Section 2, we present some lemmas and preliminaries. Section 3 discusses the existence of multiple positive solutions for BVP (1.1)-(1.2) when $f$ is positive. In Section 4, we discuss the multiplicity of positive solutions for the semipositone BVP (1.1)-(1.2). In Section 5, using the mixed monotone technique, we discuss the uniqueness of a positive solution of BVP (1.1)-(1.2).

\section{Preliminaries}

Let $C[0,1]=\{y:[0,1] \rightarrow \mathbb{R} \mid y(t)$ is continuous on $[0,1]\}$ with norm $\|y\|=\max _{t \in[0,1]}|y(t)|$. It is easy to see that $C[0,1]$ is a Banach space. Define

$$
P=\{y \in C[0,1] \mid y \text { is concave on }[0,1] \text { with } y(t) \geq 0 \text { for all } t \in[0,1]\} \text {. }
$$

It is easy to prove $P$ is a cone of $C[0,1]$.

Lemma 2.1 (see [17]) Let $\Omega$ be a bounded open set in a real Banach space E, $P$ be a cone of $E, \theta \in \Omega$ and $A: \bar{\Omega} \cap P \rightarrow P$ be continuous and compact. Suppose $\lambda A x \neq x, \forall x \in \partial \Omega \cap P$, $\lambda \in(0,1]$. Then

$$
i(A, \Omega \cap P, P)=1 .
$$

Lemma 2.2 (see [17]) Let $\Omega$ be a bounded open set in a real Banach space $E, P$ be a cone of $E, \theta \in \Omega$ and $A: \bar{\Omega} \cap P \rightarrow P$ be continuous and compact. Suppose $A x \not \leq x, \forall x \in \partial \Omega \cap P$. Then

$$
i(A, \Omega \cap P, P)=0 .
$$

Lemma 2.3 (see [18]) Let $y \in P($ defined in (2.1)). Then

$$
y(t) \geq t(1-t)\|y\| \quad \text { for } t \in[0,1] .
$$


Now we present the following conditions for convenience:

$\left(C_{1}\right) A$ and $B$ are of bounded variation with positive measures, $0<\int_{0}^{1} d A(s), a>0$, $0<\int_{0}^{1} d B(s), b>0$,

$\left(\mathrm{C}_{2}\right)$

$$
\left\{\begin{array}{l}
\text { there exists a function } \psi_{1} \\
\text { continuous on }[0,1] \text { and positive on }(0,1) \text { such that } \\
f(t, y) \geq \psi_{1}(t) \text { on }(0,1) \times(0,1]
\end{array}\right.
$$

$\left(\mathrm{C}_{3}\right)$

$$
q \in C(0,1), \quad q>0 \quad \text { on }(0,1) \text { and } \quad \int_{0}^{1} t(1-t) q(t) d t<\infty
$$

$\left(\mathrm{C}_{4}\right)$

$$
f:[0,1] \times(0, \infty) \rightarrow(0, \infty) \text { is continuous, }
$$

$\left(\mathrm{C}_{5}\right)$ there exists a continuous function $g:[0,1] \times(0, \infty) \times(0, \infty) \rightarrow(0, \infty)$ with $g(t, x, y)$ nondecreasing in $x$ and nonincreasing in $y$ and for $x>0$ we have $f(t, x)=g(t, x, x)$. Moreover, there is a constant $\theta$ with $0 \leq \theta<1$ such that

$$
g\left(t, \lambda x, \frac{1}{\lambda} y\right) \geq \lambda^{\theta} g(t, x, y), \quad \forall x>0, y>0,0<\lambda<1 .
$$

\section{Multiplicity of positive solutions for singular boundary value problems with positive nonlinearities}

In this section, we consider the existence of multiple positive solutions for BVP (1.1)-(1.2). To show that BVP (1.1)-(1.2) has a solution, for $y \in P$, define

$$
\begin{aligned}
\left(T_{\epsilon} y\right)(t)= & (1-t) \alpha[y]+t \beta[y] \\
& +\int_{0}^{1} k(t, s) q(s) f(s, \max \{\epsilon, y(s)\}) d s, \quad t \in[0,1], 1 \geq \epsilon>0,
\end{aligned}
$$

where

$$
k(t, s)= \begin{cases}(1-t) s, & 0 \leq s \leq t \leq 1 \\ (1-s) t, & 0 \leq t \leq s \leq 1\end{cases}
$$

Lemma 3.1 Suppose $\left(C_{1}\right)-\left(C_{4}\right)$ hold. Then $T_{\epsilon}: P \rightarrow P$ is continuous and completely continuous for all $1 \geq \epsilon>0$.

Proof It is easy to prove that $T_{\epsilon}$ is well defined and $\left(T_{\epsilon} y\right)(t) \geq 0$ for all $t \in P$. For $y \in P$, we have

$$
\left\{\begin{array}{l}
\left(T_{\epsilon} y\right)^{\prime \prime}(t) \leq 0 \quad \text { on }(0,1), \\
\left(T_{\epsilon} y\right)(0)=\alpha[y], \quad\left(T_{\epsilon} y\right)(1)=\beta[y]
\end{array}\right.
$$


$\left(T_{\epsilon} y\right)(t)$ is concave on $[0,1]$.

Consequently, $T_{\epsilon}: P \rightarrow P$. A standard argument shows that $T_{\epsilon}: P \rightarrow P$ is continuous and completely continuous (see $[4,19,20])$.

Lemma 3.2 Suppose that $\int_{0}^{1} d A(s)>0$ and $\int_{0}^{1} d B(s)>0$. Then

$$
\int_{0}^{1} s^{a}(1-s)^{a} d A(s)>0, \quad \int_{0}^{1} s^{b}(1-s)^{b} d B(s)>0 .
$$

The proof is trivial and we omit it.

Define

$$
\begin{aligned}
H= & \left\{x \in C([0,1], \mathbb{R}) \cap C^{1}([0,1), \mathbb{R}) \cap C((0,1),(0,+\infty)) \cap C^{2}((0,1), \mathbb{R}) \mid x\right. \text { satisfies } \\
& \left.x^{\prime \prime}(t)+q(t) f(t, \max \{\epsilon, x(t)\})=0,0<t<1, x(0)=\alpha[x], x(1)=\beta[x], \forall 1 \geq \epsilon>0\right\} .
\end{aligned}
$$

Lemma 3.3 If $H \neq \varnothing$ and $\left(\mathrm{C}_{2}\right)$ hold, there exists a $\delta_{0}>0$ such that

$$
x(t) \geq \delta_{0}, \quad \forall t \in[0,1], x \in H .
$$

Proof Suppose $x \in H$. There are two cases to consider:

(1) $\|x\|>1$. Lemma 2.3 implies that

$$
x(t) \geq t(1-t)\|x\| \geq t(1-t), \quad t \in[0,1] .
$$

(2) $0<\|x\| \leq 1$. Condition $\left(\mathrm{C}_{2}\right)$ guarantees that

$$
\begin{aligned}
x(t) & =(1-t) \alpha[x]+t \beta[x]+\int_{0}^{1} k(t, s) q(s) f(s, \max \{\epsilon, x(s)\}) d s \\
& \geq \int_{0}^{1} k(t, s) q(s) \psi_{1}(s) d s=\gamma_{0}(t), \quad t \in[0,1] .
\end{aligned}
$$

Since $\gamma_{0}^{\prime \prime}(t) \geq 0$ and $\gamma_{0}(0)=0$ and $\gamma_{0}(1)=0$, Lemma 2.3 implies that

$$
\gamma_{0}(t) \geq t(1-t)\left\|\gamma_{0}\right\|, \quad \forall t \in[0,1]
$$

Let $\delta_{1}=\min \left\{1,\left\|\gamma_{0}\right\|\right\}$. From (3.4) and (3.5), one has

$$
x(t) \geq \delta_{1} t(1-t), \quad \forall t \in[0,1] .
$$

Lemma 3.2 implies that

$$
x(0)=\alpha[x]=\int_{0}^{1} x^{a}(s) d A(s) \geq \delta_{1}^{a} \int_{0}^{1} s^{a}(1-s)^{a} d A(s)>0
$$


and

$$
x(1)=\beta[x]=\int_{0}^{1} x^{b}(s) d B(s) \geq \delta_{1}^{b} \int_{0}^{1} s^{b}(1-s)^{b} d B(s)>0 .
$$

Set

$$
\delta_{0}=\min \left\{\delta_{1}^{a} \int_{0}^{1} s^{a}(1-s)^{a} d A(s), \delta_{1}^{b} \int_{0}^{1} s^{b}(1-s)^{b} d B(s)\right\} .
$$

Since $x(t)$ is concave on $[0,1]$, we have

$$
x(t) \geq \delta_{0}, \quad \forall t \in[0,1], x \in H .
$$

The proof is complete.

Lemma 3.4 Suppose that there exists an $\bar{a} \in\left(0, \frac{1}{2}\right)$ such that

$$
\lim _{y \rightarrow+\infty} \frac{f(t, y)}{y}=+\infty
$$

uniformly on $[\bar{a}, 1-\bar{a}]$. Then there exists an $R^{\prime}>1$ such that for all $R \geq R^{\prime}$

$$
i\left(T_{\epsilon}, \Omega_{R} \cap P, P\right)=0, \quad \forall 0<\epsilon \leq 1 .
$$

Proof From (3.6), there exists a $R_{1}>1$ such that

$$
f(t, y) \geq N^{*} y, \quad \forall y \geq R_{1},
$$

where

$$
N^{*}>\frac{2}{\bar{a}^{2} \int_{\bar{a}}^{1-\bar{a}} k(\bar{a}, s) q(s) d s} .
$$

Let $R^{\prime}=\frac{R_{1}}{\bar{a}^{2}}$ and

$$
\Omega_{R}=\{x \in C[0,1] \mid\|x\|<R\}, \quad \forall R \geq R^{\prime} .
$$

Now we show

$$
T_{\epsilon} y \not \leq y \quad \text { for } y \in P \cap \partial \Omega_{R}, \forall 0<\epsilon \leq 1 \text {. }
$$

Suppose that there exists a $y_{0} \in P \cap \partial \Omega_{R}$ with $T_{\epsilon} y_{0} \leq y_{0}$. Then $\left\|y_{0}\right\|=R$. Also since $y_{0}(t)$ is concave on $[0,1]\left(\right.$ since $\left.y_{0} \in P\right)$ we have from Lemma 2.3 that $y_{0}(t) \geq t(1-t)\left\|y_{0}\right\| \geq t(1-t) R$ for $t \in[0,1]$. For $t \in[\bar{a}, 1-\bar{a}]$, one has

$$
y_{0}(t) \geq \bar{a}^{2} R \geq \bar{a}^{2} R^{\prime}=R_{1}, \quad \forall t \in[\bar{a}, 1-\bar{a}],
$$

which together with (3.7) yields the result that

$$
f\left(t, y_{0}(t)\right) \geq N^{*} y_{0}(t) \geq N^{*} \bar{a}^{2} R, \quad \forall t \in[\bar{a}, 1-\bar{a}] .
$$


Then we have, using (3.9),

$$
\begin{aligned}
y_{0}(\bar{a}) & \geq T_{\epsilon} y_{0}(\bar{a})=(1-\bar{a}) \alpha\left[y_{0}\right]+\bar{a} \beta\left[y_{0}\right]+\int_{0}^{1} k(\bar{a}, s) q(s) f\left(s, \max \left\{\epsilon, y_{0}(s)\right\}\right) d s \\
& \geq \int_{\bar{a}}^{1-\bar{a}} k(\bar{a}, s) q(s) f\left(s, \max \left\{\epsilon, y_{0}(s)\right\}\right) d s \\
& =\int_{\bar{a}}^{1-\bar{a}} k(\bar{a}, s) q(s) f\left(s, y_{0}(s)\right) d s \\
& \geq N^{*} \operatorname{R}^{2} \int_{\bar{a}}^{1-\bar{a}} k(\bar{a}, s) q(s) d s \\
& >R=\left\|y_{0}\right\|,
\end{aligned}
$$

which is a contradiction. Hence (3.8) is true. Lemma 2.2 guarantees that

$$
i\left(T_{\epsilon}, \Omega_{R} \cap P, P\right)=0, \quad \forall 0<\epsilon \leq 1
$$

The proof is complete.

Lemma 3.5 Suppose that $\max \{a, b\}>1$. Then there exists an $R^{\prime}>1$ such that for all $R \geq R^{\prime}$

$$
i\left(T_{\epsilon}, \Omega_{R} \cap P, P\right)=0, \quad \forall 0<\epsilon \leq 1
$$

Proof Since $\max \{a, b\}>1$, without loss of generality, we suppose that $a>1$. Let $R^{\prime}>1$ with $R^{\prime a-1} \int_{0}^{1} s^{a}(1-s)^{a} d A(s)>1$. Set

$$
\Omega_{R}=\{x \in C[0,1] \mid\|x\|<R\}, \quad R \geq R^{\prime} .
$$

Now we show

$$
T_{\epsilon} x \not \leq x, \quad \forall x \in \partial \Omega_{R} \cap P, \forall 0<\epsilon \leq 1 .
$$

In fact, suppose that $x_{0} \in \partial \Omega_{R} \cap P$ and satisfies

$$
T_{\epsilon} x_{0} \leq x_{0}
$$

Lemma 2.3 guarantees that

$$
x_{0}(t) \geq\left\|x_{0}\right\| t(1-t) \geq R t(1-t), \quad t \in[0,1] .
$$

Then

$$
\begin{aligned}
R & \geq x_{0}(0)=\int_{0}^{1} x_{0}^{a}(s) d A(s) \geq \int_{0}^{1}\left\|x_{0}\right\|^{a} s^{a}(1-s)^{a} d A(s) \\
& =R R^{a-1} \int_{0}^{1} s^{a}(1-s)^{a} d A(s) \geq R R^{\prime a-1} \int_{0}^{1} s^{a}(1-s)^{a} d A(s)>R .
\end{aligned}
$$


This is a contradiction. Lemma 2.2 guarantees that

$$
i\left(T_{\epsilon}, \Omega_{R} \cap P, P\right)=0, \quad \forall R \geq R^{\prime}, \forall 0<\epsilon \leq 1 .
$$

The proof is complete.

Theorem 3.1 Suppose $\left(\mathrm{C}_{1}\right),\left(\mathrm{C}_{2}\right),\left(\mathrm{C}_{3}\right)$, and $\left(\mathrm{C}_{4}\right)$ hold and the following conditions are satisfied:

$$
\left\{\begin{array}{l}
0 \leq f(t, y) \leq g(y)+h(y) \text { on }[0,1] \times(0, \infty) \text { with } \\
g>0 \text { continuous and nonincreasing on }(0, \infty), \\
h \geq 0 \text { continuous on }[0, \infty), \text { and } \frac{h}{g} \\
\text { nondecreasing on }(0, \infty)
\end{array}\right.
$$

and

$$
\sup _{r \in(0,+\infty)} \frac{1}{1+\frac{h(r)}{g(r)}} \int_{c_{0} \max \left\{r^{a}, r^{b}\right\}}^{r} \frac{d y}{g(y)}>b_{0}
$$

hold; here

$$
\begin{aligned}
& c_{0}=\max \left\{\int_{0}^{1} d A(s), \int_{0}^{1} d B(s)\right\}, \\
& b_{0}=\max \left\{2 \int_{0}^{\frac{1}{2}} t(1-t) q(t) d t, 2 \int_{\frac{1}{2}}^{1} t(1-t) q(t) d t\right\} .
\end{aligned}
$$

Then BVP (1.1)-(1.2) has at least one positive solution.

Proof Choose $\epsilon>0$ and $r>0$ with $\epsilon<\min \left\{1, c_{0}\left\{r^{a}, r^{b}\right\}\right\}$ and

$$
\frac{1}{1+\frac{h(r)}{g(r)}} \int_{c_{0}\left\{r^{a}, r^{b}\right\}}^{r} \frac{d y}{g(y)}>b_{0}
$$

Let

$$
\Omega_{1}=\{y \in C[0,1] \mid\|y\|<r\},
$$

and $T_{\epsilon}$ is defined in (3.1). Lemma 3.1 guarantees that $T_{\epsilon}: P \rightarrow P$ is continuous and completely continuous.

Now we show that

$$
y \neq \lambda T_{\epsilon} y, \quad \forall y \in \partial \Omega_{1} \cap P, \lambda \in(0,1] .
$$

Suppose that there is a $y_{0} \in \partial \Omega_{1} \cap P$ and $\lambda_{0} \in[0,1]$ with $y_{0}=\lambda_{0} T_{\epsilon} y_{0}$, i.e., $y_{0}$ satisfies

$$
\left\{\begin{array}{l}
y_{0}^{\prime \prime}+\lambda_{0} q(t) f\left(t, \max \left\{\epsilon, y_{0}(t)\right\}\right)=0, \quad 0<t<1 \\
y_{0}(0)=\lambda_{0} \alpha[y], \quad y_{0}(1)=\lambda_{0} \beta\left[y_{0}\right] .
\end{array}\right.
$$


Then $y_{0}^{\prime \prime}(t) \leq 0$ on $(0,1)$ and $y_{0}(0)=\lambda_{0} \alpha\left[y_{0}\right] \leq r^{a} \int_{0}^{1} d A(s) \leq c_{0} r^{a}<r=\left\|y_{0}\right\|, y_{0}(1)=$ $\lambda_{0} \beta\left[y_{0}\right] \leq r^{b} \int_{0}^{1} d B(s) \leq c_{0} r^{b}<r=\left\|y_{0}\right\|$, which guarantees that there exists a $t_{0} \in(0,1)$, $r=\left\|y_{0}\right\|=y_{0}\left(t_{0}\right)$ with $y^{\prime}\left(t_{0}\right)=0$ and $y_{0}^{\prime}(t) \geq 0$ for all $t \in\left(0, t_{0}\right)$ and $y_{0}^{\prime}(t) \leq 0$ for all $t \in\left(t_{0}, 1\right)$.

For $t \in(0,1)$, we have

$$
\begin{aligned}
-y_{0}^{\prime \prime}(t) & \leq g\left(\max \left\{\epsilon, y_{0}(t)\right\}\right)\left\{1+\frac{h\left(\max \left\{\epsilon, y_{0}(t)\right\}\right)}{g\left(\max \left\{\epsilon, y_{0}(t)\right\}\right)}\right\} q(t) \\
& \leq g\left(\max \left\{\epsilon, y_{0}(t)\right\}\right)\left\{1+\frac{h(r)}{g(r)}\right\} q(t) .
\end{aligned}
$$

Integrate from $t\left(t<t_{0}\right)$ to $t_{0}$ to obtain

$$
y_{0}^{\prime}(t) \leq g\left(\max \left\{\epsilon, y_{0}(t)\right\}\right)\left\{1+\frac{h(r)}{g(r)}\right\} \int_{t}^{t_{0}} q(s) d s \leq g\left(y_{0}(t)\right)\left\{1+\frac{h(r)}{g(r)}\right\} \int_{t}^{t_{0}} q(s) d s
$$

and then integrate from 0 to $t_{0}$ to obtain

$$
\begin{aligned}
\int_{\alpha\left[y_{0}\right]}^{y_{0}\left(t_{0}\right)} \frac{d y}{g(y)} & \leq\left\{1+\frac{h(r)}{g(r)}\right\} \int_{0}^{t_{0}} \int_{s}^{t_{0}} q(\tau) d \tau d s \\
& =\left\{1+\frac{h(r)}{g(r)}\right\} \int_{0}^{t_{0}} s q(s) d s \\
& \leq\left\{1+\frac{h(r)}{g(r)}\right\} \frac{1}{1-t_{0}} \int_{0}^{t_{0}} s(1-s) q(s) d s \quad\left(\text { note } 1 \leq \frac{1-s}{1-t_{0}}, \forall s \in\left[t_{0}, 1\right)\right),
\end{aligned}
$$

which together with $\alpha\left[y_{0}\right] \leq c_{0} r^{a} \leq c_{0} \max \left\{r^{a}, r^{b}\right\}$ yields the result that

$$
\int_{c_{0} \max \left\{r^{\alpha}, r^{\beta}\right\}}^{r} \frac{d y}{g(y)} \leq \int_{\alpha\left[y_{0}\right]}^{r} \frac{d y}{g(y)} \leq\left\{1+\frac{h(r)}{g(r)}\right\} \frac{1}{1-t_{0}} \int_{0}^{t_{0}} s(1-s) q(s) d s
$$

Similarly if we integrate (3.16) from $t_{0}$ to $t\left(t \geq t_{0}\right)$ and then from $t_{0}$ to 1 we obtain

$$
\int_{c_{0} \max \left\{r^{a}, r^{b}\right\}}^{r} \frac{d u}{g(u)} \leq\left\{1+\frac{h(r)}{g(r)}\right\} \frac{1}{t_{0}} \int_{t_{0}}^{1} s(1-s) q(s) d s
$$

Now (3.17) and (3.18) imply

$$
\int_{c_{0} \max \left\{r^{a}, r^{b}\right\}}^{r} \frac{d u}{g(u)} \leq b_{0}\left\{1+\frac{h(r)}{g(r)}\right\}
$$

which contradicts (3.13). Therefore, (3.14) is true. Lemma 2.1 implies that

$$
i\left(T_{\epsilon}, \Omega_{1} \cap P, P\right)=1,
$$

which yields the result that there exists a $y_{1, \epsilon} \in \Omega_{1} \cap P$ such that

$$
T_{\epsilon} y_{1, \epsilon}=y_{1, \epsilon},
$$


i.e., $H \neq \emptyset$ in Lemma 3.3. Moreover, Lemma 3.3 is true, which guarantees that there exists a $\delta_{0}>0$ such that

$$
x(t) \geq \delta_{0}, \quad \forall t \in[0,1], x \in H .
$$

Now let $\epsilon_{0}=\frac{\delta_{0}}{2}$. From the above proof, there exists a $x_{1, \epsilon_{0}} \in \Omega_{1} \cap P$ such that

$$
T_{\epsilon_{0}} x_{1, \epsilon_{0}}=x_{1, \epsilon_{0}}
$$

Since $0<\epsilon_{0} \leq 1,(3.20)$ implies that

$$
x_{1, \epsilon_{0}}(t) \geq \delta_{0}>\epsilon_{0}, \quad t \in[0,1] .
$$

Moreover, since $x_{1, \epsilon_{0}}(t)$ satisfies

$$
\left\{\begin{array}{l}
x_{1, \epsilon_{0}}^{\prime \prime}(t)+q(t) f\left(t, \max \left\{\epsilon_{0}, x_{1, \epsilon_{0}}(t)\right\}\right)=0, \quad 0<t<1, \\
x_{1, \epsilon_{0}}(0)=\alpha\left[x_{1, \epsilon_{0}}\right], \quad x_{1, \epsilon_{0}}(1)=\beta\left[x_{1, \epsilon_{0}}\right],
\end{array}\right.
$$

(3.21) guarantees that

$$
\left\{\begin{array}{l}
x_{1, \epsilon_{0}}^{\prime \prime}(t)+q(t) f\left(t, x_{1, \epsilon_{0}}(t)\right)=0, \quad 0<t<1, \\
x_{1, \epsilon_{0}}(0)=\alpha\left[x_{1, \epsilon_{0}}\right], \quad x_{1, \epsilon_{0}}(1)=\beta\left[x_{1, \epsilon_{0}}\right] .
\end{array}\right.
$$

Thus, BVP (1.1)-(1.2) has at least one positive solution. The proof is complete.

Theorem 3.2 Suppose the conditions of Theorem 3.1 hold and there exists an $\bar{a} \in\left(0, \frac{1}{2}\right)$ such that

$$
\lim _{y \rightarrow+\infty} \frac{f(t, y)}{y}=+\infty
$$

uniformly on $[\bar{a}, 1-\bar{a}]$. Then BVP (1.1)-(1.2) has at least two positive solutions.

Proof Choose $r>0$ as in (3.13), $\epsilon_{0}>0$ with $\epsilon_{0}<\min \left\{\delta_{0}, 1, c_{0}\left\{r^{a}, r^{b}\right\}\right\}$, where $\delta_{0}$ is defined in (3.20), and $R>\max \left\{r, R^{\prime}\right\}$ in Lemma 3.4. Set

$$
\begin{aligned}
& \Omega_{1}=\{y \in C[0,1] \mid\|y\|<r\}, \\
& \Omega_{2}=\{y \in C[0,1] \mid\|y\|<R\} .
\end{aligned}
$$

From the proof of Theorem 3.1 and Lemma 3.4, we have

$$
i\left(T_{\epsilon_{0}}, \Omega_{1} \cap P, P\right)=1
$$

and

$$
i\left(T_{\epsilon_{0}}, \Omega_{2} \cap P, P\right)=0,
$$


which implies that

$$
i\left(T_{\epsilon_{0}},\left(\Omega_{2}-\Omega_{1}\right) \cap P, P\right)=-1 .
$$

Thus, there exist $x_{1, \epsilon_{0}} \in \Omega_{1} \cap P$ and $x_{2, \epsilon_{0}} \in\left(\Omega_{2}-\Omega_{1}\right) \cap P$ such that

$$
T_{\epsilon_{0}} x_{1, \epsilon_{0}}=x_{1, \epsilon_{0}}, \quad T_{\epsilon_{0}} x_{2, \epsilon_{0}}=x_{2, \epsilon_{0}} .
$$

From the proof of Theorem 3.1, $x_{1, \epsilon_{0}}(t)$ and $x_{2, \epsilon_{0}}(t)$ are two positive solutions for BVP (1.1)-(1.2). The proof is complete.

Theorem 3.3 Suppose the conditions of Theorem 3.1 hold and $\max \{a, b\}>1$. Then BVP (1.1)-(1.2) has at least two positive solutions.

Proof Choose $r>0$ as in (3.13), $\epsilon_{0}>0$ with $\epsilon_{0}<\min \left\{\delta_{0}, 1, c_{0}\left\{r^{a}, r^{b}\right\}\right\}$, where $\delta_{0}$ is defined in (3.20), and $R>\max \left\{r, R^{\prime}\right\}$ in Lemma 3.5. Set

$$
\begin{aligned}
& \Omega_{1}=\{y \in C[0,1] \mid\|y\|<r\}, \\
& \Omega_{2}=\{y \in C[0,1] \mid\|y\|<R\} .
\end{aligned}
$$

From the proof of Theorem 3.1 and Lemma 3.5, we have

$$
i\left(T_{\epsilon_{0}}, \Omega_{1} \cap P, P\right)=1
$$

and

$$
i\left(T_{\epsilon_{0}}, \Omega_{2} \cap P, P\right)=0,
$$

which implies that

$$
i\left(T_{\epsilon_{0}},\left(\Omega_{2}-\Omega_{1}\right) \cap P, P\right)=-1 .
$$

Thus, there exist $x_{1, \epsilon_{0}} \in \Omega_{1} \cap P$ and $x_{2, \epsilon_{0}} \in\left(\Omega_{2}-\Omega_{1}\right) \cap P$ such that

$$
T_{\epsilon_{0}} x_{1, \epsilon_{0}}=x_{1, \epsilon_{0}}, \quad T_{\epsilon_{0}} x_{2, \epsilon_{0}}=x_{2, \epsilon_{0}} .
$$

From the proof of Theorem 3.1, $x_{1, \epsilon_{0}}(t)$ and $x_{2, \epsilon_{0}}(t)$ are two positive solutions for BVP (1.1)-(1.2). The proof is complete.

Example 3.1 Consider

$$
y^{\prime \prime}(t)+\mu\left(y^{-\delta_{1}}(t)+y^{\delta_{2}}(t)\right)=0, \quad 0<t<1,
$$

with

$$
y(0)=\int_{0}^{1} y^{\frac{1}{3}}(s) d A(s), \quad y(1)=\int_{0}^{1} y^{\frac{1}{2}}(s) d B(s), \quad d A(s)=\frac{1}{2} d s, d B(s)=\frac{1}{8} d e^{s},
$$

where $\delta_{1}>0, \delta_{2}>1$. 
Let $q(t)=\mu, f(t, y)=y^{-\delta_{1}}+y^{\delta_{2}}, g(y)=y^{-\delta_{1}}, h(y)=y^{\delta_{2}}, c_{0}=\max \left\{\int_{0}^{1} d A(s), \int_{0}^{1} d B(s)\right\}=\frac{1}{2}$, $b_{0}=\frac{1}{6} \mu$. It is easy to see that $\left(C_{1}\right)-\left(C_{4}\right)$ and (3.10) hold. Since

$$
\frac{1}{1+\frac{h(1)}{g(1)}} \int_{c_{0} \max \left\{1^{\frac{1}{3}, 1^{\frac{1}{2}}}\right.}^{1} \frac{1}{g(y)} d y=\frac{1-\left(\frac{1}{2}\right)^{\delta_{1}+1}}{2\left(1+\delta_{1}\right)}
$$

letting $\mu_{0}<3 \frac{1-\left(\frac{1}{2}\right)^{\delta_{1}+1}}{2\left(1+\delta_{1}\right)}$, we have

$$
\sup _{r \in(0,+\infty)} \frac{1}{1+\frac{h(r)}{g(r)}} \int_{c_{0} \max \left\{r^{\frac{1}{3}}, r^{\frac{1}{2}}\right\}}^{r} \frac{1}{g(y)} d y>b_{0}
$$

for all $\mu \leq \mu_{0}$, which guarantees that (3.11) is true. Moreover, since

$$
\lim _{y \rightarrow+\infty} \frac{f(t, y)}{y}=+\infty
$$

uniformly on $[0,1]$, all the conditions of Theorem 3.2 hold, which implies that (3.22)-(3.23) has at least two positive solutions (for $\mu \leq \mu_{0}$ ).

Example 3.2 Consider

$$
y^{\prime \prime}(t)+\mu\left(y^{-\delta_{1}}(t)+y^{\delta_{2}}(t)\right)=0, \quad 0<t<1
$$

with

$$
y(0)=\int_{0}^{1} y^{3}(s) d A(s), \quad y(1)=\int_{0}^{1} y^{\frac{1}{2}}(s) d B(s), \quad d A(s)=\frac{1}{2} d s, d B(s)=\frac{1}{8} d e^{s},
$$

where $\delta_{1}>0, \delta_{2}<1$.

It is easy to see that all conditions of Theorem 3.3 hold, which implies that (3.24)-(3.25) has at least two positive solutions.

\section{Multiplicity of positive solutions for the singular semipositone boundary value problem}

In this section, we consider the case

$$
f(t, y)=F(t, y)-\gamma(t), \quad t \in(0,1)
$$

where the conditions $\left(C_{1}\right),\left(C_{3}\right),\left(C_{4}\right)$ for $F(t, y)$ instead of $f(t, y)$ hold and $\gamma \in C((0,1)$, $(0,+\infty))$ with

$$
w(t)=\int_{0}^{1} k(t, s) q(s) \gamma(s) d s<+\infty, \quad t \in[0,1], \quad c_{1}=\int_{0}^{1} q(t) \gamma(t) d t<+\infty
$$

For $y \in P$, define

$$
\begin{aligned}
\left(T_{\epsilon} y\right)(t)= & (1-t) \alpha\left[[y-w]^{*}\right]+t \beta\left[[y-w]^{*}\right] \\
& +\int_{0}^{1} k(t, s) q(s) F\left(s, \max \left\{\epsilon,[y(s)-w(s)]^{*}\right\}\right) d s, \quad t \in[0,1], 0<\epsilon \leq 1,
\end{aligned}
$$


where $k(t, s)$ is defined in (3.1) and

$$
[y(t)-w(t)]^{*}= \begin{cases}y(t)-w(t), & \text { if } y(t)-w(t)>0 \\ 0, & \text { if } y(t)-w(t) \leq 0\end{cases}
$$

Now we present the following condition for convenience:

$\left(\mathrm{C}_{2}\right)^{\prime}$

$$
\left\{\begin{array}{l}
\text { there exists a function } \psi_{4 c_{1}} \\
\text { continuous on }[0,1] \text { and positive on }(0,1) \text { such that } \\
F(t, y) \geq \psi_{4 c_{1}}(t) \text { on }(0,1) \times\left(0,4 c_{1}\right] \text { with } \\
\max _{t \in[0,1]} \int_{0}^{1} k(t, s) q(s) \psi_{4 c_{1}}(s) d s>2 c_{1}
\end{array}\right.
$$

Define

$$
\begin{aligned}
H= & \left\{x \in C([0,1], \mathbb{R}) \cap C^{1}([0,1), \mathbb{R}) \cap C((0,1),(0,+\infty)) \cap C^{2}((0,1), \mathbb{R}) \mid x\right. \text { satisfies } \\
& x^{\prime \prime}(t)+q(t) F\left(t, \max \left\{\epsilon,[x(t)-w(t)]^{*}\right\}\right)=0,0<t<1, \\
& \left.x(0)=\alpha\left[[x-w]^{*}\right], x(1)=\beta\left[[x-w]^{*}\right], \forall 1 \geq \epsilon>0\right\} .
\end{aligned}
$$

Lemma 4.1 If $H \neq \emptyset$ and $\left(\mathrm{C}_{2}\right)^{\prime}$ hold, then there exists $a \delta_{0}>0$ such that

$$
[x(t)-w(t)]^{*} \geq \delta_{0}, \quad \forall t \in[0,1], x \in H .
$$

Proof Suppose that $x \in H$. There are two cases to consider:

(1) $\|x\| \geq 4 c_{1}$. Since

$$
w(t) \leq t(1-t) \int_{0}^{1} q(s) \gamma(s) d s=c_{1} t(1-t),
$$

we have

$$
w(t) \leq \frac{1}{4} 4 c_{1} t(1-t) \leq \frac{1}{4}\|x\| t(1-t) .
$$

From Lemma 2.3, we have

$$
x(t) \geq\|x\| t(1-t) \geq 4 c_{1} t(1-t), \quad \forall t \in(0,1),
$$

which implies that

$$
\begin{aligned}
x(t)-w(t) & \geq\|x\| t(1-t)-\frac{1}{4}\|x\| t(1-t)=\frac{3}{4}\|x\| t(1-t) \\
& \geq \frac{3}{4} 4 c_{1} t(1-t)=3 c_{1} t(1-t), \quad t \in[0,1] .
\end{aligned}
$$

Hence

$$
[x(t)-w(t)]^{*} \geq 3 c_{1} t(1-t), \quad t \in[0,1]
$$


and so

$$
x(0)=\alpha\left[[x-w]^{*}\right] \geq \int_{0}^{1}\left(3 c_{1} s(1-s)\right)^{a} d A(s), x(1)=\beta\left[[x-w]^{*}\right] \geq \int_{0}^{1}\left(3 c_{1} s(1-s)\right)^{b} d B(s) .
$$

The concavity of $x(t)$ implies that

$$
x(t) \geq \min \left\{\int_{0}^{1}\left(3 c_{1} s(1-s)\right)^{a} d A(s), \int_{0}^{1}\left(3 c_{1} s(1-s)\right)^{b} d B(s)\right\} \stackrel{\text { def. }}{=} \delta_{1} .
$$

Then

$$
[x(t)-w(t)]^{*} \geq \frac{3}{4} x(t) \geq \frac{3}{4} \delta_{1}, \quad t \in[0,1] .
$$

(2) $0<\|x\| \leq 4 c_{1}$. Condition $\left(C_{2}\right)^{\prime}$ guarantees that

$$
\begin{aligned}
\|x\| & \geq \max _{t \in[0,1]} \int_{0}^{1} k(t, s) q(s) F\left(s, \max \left\{\epsilon,[x(s)-w(s)]^{*}\right\}\right) d s \\
& \geq \max _{t \in[0,1]} \int_{0}^{1} k(t, s) q(s) \psi_{4 c_{1}}(s) d s>2 c_{1},
\end{aligned}
$$

which together with $x \in P$ implies that

$$
x(t) \geq t(1-t)\|x\| \geq 2 c_{1} t(1-t), \quad t \in[0,1] .
$$

From (4.2) and (4.4), we have

$$
w(t) \leq c_{1} t(1-t)=\frac{1}{2} 2 c_{1} t(1-t) \leq \frac{1}{2} x(t), \quad t \in[0,1],
$$

and so

$$
[x(t)-w(t)]^{*} \geq \frac{1}{2} x(t) \geq c_{1} t(1-t), \quad t \in[0,1] .
$$

Then

$$
\begin{aligned}
x(t) & =(1-t) \alpha\left[[x-w]^{*}\right]+t \beta\left[[x-w]^{*}\right]+\int_{0}^{1} k(t, s) q(s) F\left(s, \max \left\{\epsilon,[x(s)-w(s)]^{*}\right\}\right) d s \\
& \geq(1-t) \int_{0}^{1}\left(c_{1} s(1-s)\right)^{a} d A(s)+t \int_{0}^{1}\left(c_{1} s(1-s)\right)^{b} d B(s) \\
& \geq \min _{t \in[0,1]}\left[(1-t) \int_{0}^{1}\left(c_{1} s(1-s)\right)^{a} d A(s)+t \int_{0}^{1}\left(c_{1} s(1-s)\right)^{b} d B(s)\right], \quad t \in[0,1],
\end{aligned}
$$

which implies

$$
\begin{aligned}
{[x(t)-w(t)]^{*} } & \geq \frac{1}{2} x(t) \\
& \geq \frac{1}{2} \min _{t \in[0,1]}\left[(1-t) \int_{0}^{1}\left(c_{1} s(1-s)\right)^{a} d A(s)+t \int_{0}^{1}\left(c_{1} s(1-s)\right)^{b} d B(s)\right] \\
& \stackrel{\text { def. }}{=} \delta_{2} .
\end{aligned}
$$


Let

$$
\delta_{0}=\min \left\{\delta_{2}, \frac{3}{4} \delta_{1}\right\} .
$$

Now (4.3) and (4.5) guarantee that

$$
[x(t)-w(t)]^{*} \geq \delta_{0}, \quad t \in[0,1] .
$$

The proof is complete.

Lemma 4.2 Suppose there exists an $\bar{a} \in\left(0, \frac{1}{2}\right)$ such that

$$
\lim _{y \rightarrow+\infty} \frac{F(t, y)}{y}=+\infty
$$

uniformly on $[\bar{a}, 1-\bar{a}]$. Then there exists an $R^{\prime}>0$ such that for all $R \geq R^{\prime}$

$$
i\left(T_{\epsilon}, \Omega_{R} \cap P, P\right)=0, \quad \forall 0<\epsilon \leq 1 .
$$

Proof From (4.6), there exists a $R_{1}>\max \left\{1,2 c_{1}\right\}$ such that

$$
F(t, y) \geq N^{*} y, \quad \forall y \geq R_{1},
$$

where

$$
N^{*}>\frac{2}{\bar{a}^{2} \int_{\bar{a}}^{1-\bar{a}} k(\bar{a}, s) q(s) d s} .
$$

Let $R^{\prime}=\frac{2}{\bar{a}^{2}} R_{1}$ and

$$
\Omega_{R}=\{x \in C[0,1] \mid\|x\|<R\}, \quad R \geq R^{\prime} .
$$

Now we show

$$
T_{\epsilon} y \not \leq y \quad \text { for } y \in P \cap \partial \Omega_{R}, 0<\epsilon \leq 1 \text {. }
$$

Suppose that there exists a $y_{0} \in P \cap \partial \Omega_{R}$ with $T_{\epsilon} y_{0} \leq y_{0}$. Then $\left\|y_{0}\right\|=R$. Also since $y_{0}(t)$ is concave on $[0,1]$ (since $y_{0} \in P$ ) we have from Lemma 2.3 that $y_{0}(t) \geq t(1-t)\left\|y_{0}\right\| \geq t(1-t) R$ for $t \in[0,1]$. For $t \in[\bar{a}, 1-\bar{a}]$, we have (notice $\left\|y_{0}\right\|=R \geq 2 c_{1}$ )

$$
\left[y_{0}(t)-w(t)\right]^{*} \geq \frac{1}{2} y_{0}(t) \geq \frac{1}{2} R \bar{a}^{2} \geq R_{1}, \quad \forall t \in[\bar{a}, 1-\bar{a}],
$$

which together with (4.7) yields the result that

$$
F\left(t, \max \left\{\epsilon,\left[y_{0}(t)-w(t)\right]^{*}\right\}\right) \geq N^{*}\left[y_{0}(t)-w(t)\right]^{*} \geq N^{*} \frac{1}{2} \operatorname{Ra}^{2}, \quad \forall t \in[\bar{a}, 1-\bar{a}] .
$$


Then we have, using (4.9),

$$
\begin{aligned}
y_{0}(\bar{a}) \geq & T_{\epsilon} y_{0}(\bar{a}) \\
= & (1-\bar{a}) \alpha\left[\left[y_{0}-w\right]^{*}\right]+\bar{a} \beta\left[\left[y_{0}-w\right]^{*}\right] \\
& +\int_{0}^{1} k(\bar{a}, s) q(s) F\left(s, \max \left\{\epsilon,\left[y_{0}(s)-w(s)\right]^{*}\right\}\right) d s \\
\geq & \int_{\bar{a}}^{1-\bar{a}} k(\bar{a}, s) q(s) F\left(s, \max \left\{\epsilon,\left[y_{0}(s)-w(s)\right]^{*}\right\}\right) d s \\
= & \int_{\bar{a}}^{1-\bar{a}} k(\bar{a}, s) q(s) F\left(s,\left[y_{0}(s)-w(s)\right]^{*}\right) d s \\
\geq & N^{*} \frac{1}{2} R \bar{a}^{2} \int_{\bar{a}}^{1-\bar{a}} k(\bar{a}, s) q(s) d s>R=\left\|y_{0}\right\|,
\end{aligned}
$$

which is a contradiction. Hence (4.8) is true. Thus Lemma 2.2 guarantees that

$$
i\left(T_{\epsilon}, \Omega_{R} \cap P, P\right)=0, \quad \forall 0<\epsilon \leq 1 .
$$

The proof is complete.

Lemma 4.3 Suppose that $\max \{a, b\}>1$. Then there exists an $R^{\prime}>0$ such that for all $R \geq R^{\prime}$

$$
i\left(T_{\epsilon}, \Omega_{R} \cap P, P\right)=0, \quad \forall 0<\epsilon \leq 1 .
$$

Proof Since $\max \{a, b\}>1$, without loss of generality, we suppose that $a>1$. Let $R^{\prime}>$ $\max \left\{1,2 c_{1}\right\}$ with $\frac{1}{2^{a}} R^{\prime a-1} \int_{0}^{1} s^{a}(1-s)^{a} d A(s)>1$. Set

$$
\Omega_{R}=\{x \in C[0,1] \mid\|x\|<R\} .
$$

Now we show that

$$
T_{\epsilon} x \not \leq x, \quad \forall x \in \partial \Omega_{R} \cap P, \forall 0<\epsilon \leq 1 .
$$

In fact, suppose that $x_{0} \in \partial \Omega_{R} \cap P$ and satisfies

$$
T_{\epsilon} x \leq x
$$

Then $\left\|y_{0}\right\|=R$. Also since $y_{0}(t)$ is concave on $[0,1]$ (since $y_{0} \in P$ ) we have from Lemma 2.3 that $y_{0}(t) \geq t(1-t)\left\|y_{0}\right\| \geq t(1-t) R$ for $t \in[0,1]$. For $t \in[0,1]$ we have

$$
\left[y_{0}(t)-w(t)\right]^{*} \geq \frac{1}{2} y_{0}(t) \geq \frac{1}{2} R t(1-t) .
$$

Then

$$
\begin{aligned}
R & \geq y(0)=\int_{0}^{1}\left(\left[y_{0}(s)-w(s)\right]^{*}\right)^{a} d A(s) \geq \int_{0}^{1}\left(\frac{1}{2}\left\|y_{0}\right\| s(1-s)\right)^{a} d A(s) \\
& =\frac{1}{2^{a}} R R^{a-1} \int_{0}^{1} s^{a}(1-s)^{a} d A(s) \geq \frac{1}{2^{a}} R R^{\prime a-1} \int_{0}^{1} s^{a}(1-s)^{a} d A(s)>R .
\end{aligned}
$$


This is a contradiction. Lemma 2.3 guarantees that

$$
i\left(T_{\epsilon}, \Omega_{R} \cap P, P\right)=0, \quad \forall 0<\epsilon \leq 1 .
$$

The proof is complete.

Theorem 4.1 Suppose $\left(\mathrm{C}_{1}\right),\left(\mathrm{C}_{2}\right)^{\prime},\left(\mathrm{C}_{3}\right)$, and $\left(\mathrm{C}_{4}\right)$ hold and the following conditions are satisfied:

$$
\left\{\begin{array}{l}
0 \leq F(t, y) \leq g(y)+h(y) \text { on }[0,1] \times(0, \infty) \text { with } \\
g>0 \text { continuous and nonincreasing on }(0, \infty), \\
h \geq 0 \text { continuous on }[0, \infty), \text { and } \frac{h}{g} \\
\text { nondecreasing on }(0, \infty),
\end{array}\right.
$$

and

$$
\sup _{r \in\left(2 c_{1},+\infty\right)} \frac{1}{1+\frac{h(r)}{g(r)}} \int_{c_{0} \max \left\{r^{a}, r^{b}\right\}}^{r} \frac{d y}{g\left(\frac{1}{2} y\right)}>b_{0}
$$

holds; here

$$
\begin{aligned}
& c_{0}=\max \left\{\int_{0}^{1} d A(s), \int_{0}^{1} d B(s)\right\}, \\
& b_{0}=\max \left\{2 \int_{0}^{\frac{1}{2}} t(1-t) q(t) d t, 2 \int_{\frac{1}{2}}^{1} t(1-t) q(t) d t\right\} .
\end{aligned}
$$

Then BVP (1.1)-(1.2) has at least one positive solution.

Proof From (4.11), choose $r>2 c_{1}, \epsilon>0$ with $\epsilon<\min \left\{1, \frac{1}{2} c_{0} \max \left\{r^{a}, r^{b}\right\}\right\}$ with

$$
\frac{1}{1+\frac{h(r)}{g(r)}} \int_{c_{0} \max \left\{r^{a}, r^{b}\right\}}^{r} \frac{d y}{g\left(\frac{1}{2} y\right)}>b_{0} .
$$

Let

$$
\Omega_{1}=\{y \in C[0,1] \mid\|y\|<r\} .
$$

Let $T_{\epsilon}$ be defined as in (4.1). Lemma 3.1 guarantees that $T_{\epsilon}: P \rightarrow P$ is continuous and completely continuous.

Now we show that

$$
y \neq \lambda T_{\epsilon} y, \quad \forall y \in \partial \Omega_{1} \cap P, \lambda \in[0,1] .
$$

Suppose that there is a $y_{0} \in \partial \Omega_{1} \cap P$ and $\lambda_{0} \in[0,1]$ with $y_{0}=\lambda_{0} T_{\epsilon} y_{0}$. Since $y_{0}(t) \geq$ $t(1-t)\left\|y_{0}\right\| \geq t(1-t) 2 c_{1}$ and $w(t)=\int_{0}^{1} k(t, s) q(s) \gamma(s) d s \leq t(1-t) \int_{0}^{1} q(s) \gamma(s) d s=c_{1} t(1-t)=$ $\frac{c_{1}}{\left\|y_{0}\right\|} t(1-t)\left\|y_{0}\right\| \leq \frac{1}{2} y_{0}(t)$, we have

$$
y_{0}(t)-w(t) \geq \frac{1}{2} y_{0}(t), \quad t \in[0,1] .
$$


Since $y_{0}$ satisfies

$$
\left\{\begin{array}{l}
y_{0}^{\prime \prime}+\lambda_{0} q(t) F\left(t, \max \left\{\epsilon,\left[y_{0}(t)-w(t)\right]^{*}\right\}\right)=0, \quad 0<t<1, \\
y_{0}(0)=\lambda_{0} \alpha\left[\left[y_{0}-w\right]^{*}\right], \quad y_{0}(1)=\lambda_{0} \beta\left[\left[y_{0}-w\right]^{*}\right]
\end{array}\right.
$$

$y_{0}(0)=\lambda_{0} \alpha\left[\left[y_{0}-w\right]^{*}\right] \leq r^{a} \int_{0}^{1} d A(s) \leq c_{0} r^{a}<r=\left\|y_{0}\right\|$ and $y_{0}(1)=\lambda_{0} \beta\left[\left[y_{0}-w\right]^{*}\right] \leq$ $r^{b} \int_{0}^{1} d B(s) \leq c_{0} r^{b}<r=\left\|y_{0}\right\|$, there exists a $t_{0} \in(0,1)$ such that $y_{0}^{\prime}\left(t_{0}\right)=0$ and $y_{0}^{\prime}(t) \geq 0$ on $\left(0, t_{0}\right), y_{0}^{\prime}(t) \leq 0$ on $\left(t_{0}, 1\right)$. For $t \in(0,1)$, it is easy to see that

$$
\begin{aligned}
-y_{0}^{\prime \prime}(t) & \leq g\left(\max \left\{\epsilon,\left[y_{0}(t)-w(t)\right]^{*}\right\}\right)\left\{1+\frac{h\left(\max \left\{\epsilon,\left[y_{0}(t)-w(t)\right]^{*}\right\}\right)}{g\left(\max \left\{\epsilon,\left[y_{0}(t)-w(t)\right]^{*}\right\}\right)}\right\} q(t) \\
& \leq g\left(\max \left\{\epsilon,\left[y_{0}(t)-w(t)\right]^{*}\right\}\right)\left\{1+\frac{h(r)}{g(r)}\right\} q(t) .
\end{aligned}
$$

Integrate from $t$ to $t_{0}$ to obtain

$$
\begin{aligned}
y_{0}^{\prime}(t) & \leq g\left(\max \left\{\epsilon,\left[y_{0}(t)-w(t)\right]^{*}\right\}\right)\left\{1+\frac{h(r)}{g(r)}\right\} \int_{t}^{t_{0}} q(s) d s \\
& \leq g\left(\max \left\{\epsilon, \frac{1}{2} y_{0}(t)\right\}\right)\left\{1+\frac{h(r)}{g(r)}\right\} \int_{t}^{t_{0}} q(s) d s \\
& \leq g\left(\frac{1}{2} y_{0}(t)\right)\left\{1+\frac{h(r)}{g(r)}\right\} \int_{t}^{t_{0}} q(s) d s
\end{aligned}
$$

and then integrate from 0 to $t_{0}$ to obtain

$$
\int_{\alpha\left[\left[y_{0}-w\right]^{*}\right]}^{y_{0}\left(t_{0}\right)} \frac{d y}{g\left(\frac{1}{2} y\right)} \leq\left\{1+\frac{h(r)}{g(r)}\right\} \int_{0}^{t_{0}} s q(s) d s \leq\left\{1+\frac{h(r)}{g(r)}\right\} \frac{1}{1-t_{0}} \int_{0}^{t_{0}} s(1-s) d s,
$$

which together with $\alpha\left[\left[y_{0}-w\right]^{*}\right] \leq \alpha\left[y_{0}\right] \leq c_{0} \max \left\{r^{a}, r^{b}\right\}$ yields

$$
\int_{c_{0} \max \left\{r^{a}, r^{\beta}\right\}}^{r} \frac{d y}{g\left(\frac{1}{2} y\right)} \leq \int_{\alpha\left[\left[y_{0}-w\right]^{*}\right]}^{r} \frac{d y}{g\left(\frac{1}{2} y\right)} \leq\left\{1+\frac{h(r)}{g(r)}\right\} \frac{1}{1-t_{0}} \int_{0}^{t_{0}} s(1-s) d s .
$$

Similarly if we integrate (4.16) from $t_{0}$ to $t\left(t \geq t_{0}\right)$ and then from $t_{0}$ to 1 we obtain

$$
\int_{c_{0} \max \left\{r^{a}, r^{b}\right\}}^{r} \frac{d u}{g\left(\frac{1}{2} u\right)} \leq\left\{1+\frac{h(r)}{g(r)}\right\} \frac{1}{t_{0}} \int_{t_{0}}^{1} s(1-s) q(s) d s .
$$

Now (4.17) and (4.18) imply

$$
\int_{c_{0} \max \left\{r^{a}, r^{b}\right\}}^{r} \frac{d u}{g\left(\frac{1}{2} u\right)} \leq b_{0}\left\{1+\frac{h(r)}{g(r)}\right\}
$$

which contradicts (4.13). Then (4.14) is true. Lemma 2.1 implies that

$$
i\left(T_{\epsilon}, \Omega_{1} \cap P, P\right)=1 \text {. }
$$


Thus, there exists an $x_{\epsilon} \in P \cap \Omega_{1}$ such that $T_{\epsilon} \mathcal{X}_{\epsilon}=x_{\epsilon}$, which yields the result that $H \neq \emptyset$ in Lemma 4.1 and there is a $\delta_{0}>0$ such that

$$
[x(t)-w(t)]^{*} \geq \delta_{0}, \quad \forall x \in H .
$$

Let $\epsilon_{0}=\frac{1}{2} \delta_{0}$ and $T_{\epsilon_{0}} x_{\epsilon_{0}}=x_{\epsilon_{0}}$. Obviously $x_{\epsilon_{0}} \in H$ and $\left[x_{\epsilon_{0}}(t)-w(t)\right]^{*} \geq \delta_{0}$. From

$$
x_{\epsilon_{0}}(t)=(1-t) \alpha\left[\left[x_{\epsilon_{0}}-w\right]^{*}\right]+t \beta\left[\left[x_{\epsilon_{0}}-w\right]^{*}\right]+\int_{0}^{1} k(t, s) q(s) F\left(s,\left[\left[x_{\epsilon_{0}}(s)-w(s)\right]^{*}\right]\right) d s,
$$

we have

$$
x_{\epsilon_{0}}(t)=(1-t) \alpha\left[x_{\epsilon_{0}}-w\right]+t \beta\left[x_{\epsilon_{0}}-w\right]+\int_{0}^{1} k(t, s) q(s) F\left(s, x_{\epsilon_{0}}(s)-w(s)\right) d s, \quad t \in[0,1] .
$$

Let $y(t)=x_{\epsilon_{0}}(t)-w(t), t \in[0,1]$. It is easy to see that $y(t)$ is a positive solution of BVP (1.1)(1.2). The proof is complete.

Theorem 4.2 Suppose the conditions of Theorem 4.1 hold and there exists an $\bar{a} \in\left(0, \frac{1}{2}\right)$ such that

$$
\lim _{y \rightarrow+\infty} \frac{F(t, y)}{y}=+\infty
$$

uniformly on $[\bar{a}, 1-\bar{a}]$. Then BVP (1.1)-(1.2) has at least two positive solutions.

Proof Choose $r$ as in (4.13), $\epsilon_{0}>0$ with $\epsilon_{0}<\min \left\{\delta_{0}, 1, \frac{1}{2} c_{0} \max \left\{r^{a}, r^{b}\right\}\right\}$, where $\delta_{0}$ is defined in (4.20), and $R>\max \{1, r\}$ in Lemma 4.2. Set

$$
\begin{aligned}
& \Omega_{1}=\{y \in C[0,1] \mid\|y\|<r\}, \\
& \Omega_{2}=\{y \in C[0,1] \mid\|y\|<R\} .
\end{aligned}
$$

From the proof of Theorem 4.1 and Lemma 4.2, we have

$$
i\left(T_{\epsilon_{0}}, \Omega_{1} \cap P, P\right)=1
$$

and

$$
i\left(T_{\epsilon_{0}}, \Omega_{2} \cap P, P\right)=0,
$$

which implies that

$$
i\left(T_{\epsilon_{0}},\left(\Omega_{2}-\Omega_{1}\right) \cap P, P\right)=-1 .
$$

Thus, there exist $x_{1, \epsilon_{0}} \in \Omega_{1} \cap P$ and $x_{2, \epsilon_{0}} \in\left(\Omega_{2}-\Omega_{1}\right) \cap P$ such that

$$
T_{\epsilon_{0}} x_{1, \epsilon_{0}}=x_{1, \epsilon_{0}}, \quad T_{\epsilon_{0}} x_{2, \epsilon_{0}}=x_{2, \epsilon_{0}} .
$$


Let $y_{1, \epsilon_{0}}(t)=x_{1, \epsilon_{0}}(t)-w(t)$ and $y_{2, \epsilon_{0}}(t)=x_{2, \epsilon_{0}}(t)-w(t)$ for all $t \in[0,1]$. It is easy to see that $y_{1, \epsilon_{0}}(t)$ and $y_{2, \epsilon_{0}}(t)$ are two positive solutions for BVP (1.1)-(1.2). The proof is complete.

Theorem 4.3 Suppose the conditions of Theorem 4.1 hold and $\max \{a, b\}>1$. Then BVP (1.1)-(1.2) has at least two positive solutions.

Proof Choose $r$ as in (4.13), $\epsilon_{0}>0$ with $\epsilon_{0}<\min \left\{\delta_{0}, 1, \frac{1}{2} c_{0} \max \left\{r^{a}, r^{b}\right\}\right\}$, where $\delta_{0}$ is defined in (4.20), and $R>\max \{1, r\}$ in Lemma 4.3. Set

$$
\begin{aligned}
& \Omega_{1}=\{y \in C[0,1] \mid\|y\|<r\}, \\
& \Omega_{2}=\{y \in C[0,1] \mid\|y\|<R\} .
\end{aligned}
$$

From the proof of Theorem 4.1 and Lemma 4.3, we have

$$
i\left(T_{\epsilon_{0}}, \Omega_{1} \cap P, P\right)=1
$$

and

$$
i\left(T_{\epsilon_{0}}, \Omega_{2} \cap P, P\right)=0,
$$

which implies that

$$
i\left(T_{\epsilon_{0}},\left(\Omega_{2}-\Omega_{1}\right) \cap P, P\right)=-1 .
$$

Thus, there exist $x_{1, \epsilon_{0}} \in \Omega_{1} \cap P$ and $x_{2, \epsilon_{0}} \in\left(\Omega_{2}-\Omega_{1}\right) \cap P$ such that

$$
T_{\epsilon_{0}} x_{1, \epsilon_{0}}=x_{1, \epsilon_{0}}, \quad T_{\epsilon_{0}} x_{2, \epsilon_{0}}=x_{2, \epsilon_{0}} .
$$

Let $y_{1, \epsilon_{0}}(t)=x_{1, \epsilon_{0}}(t)-w(t)$ and $y_{2, \epsilon_{0}}(t)=x_{2, \epsilon_{0}}(t)-w(t)$ for all $t \in[0,1]$. It is easy to see that $y_{1, \epsilon_{0}}(t)$ and $y_{2, \epsilon_{0}}(t)$ are two positive solutions for BVP (1.1)-(1.2). The proof is complete.

Example 4.1 Consider

$$
\begin{aligned}
& y^{\prime \prime}(t)+\frac{7}{64}\left(y^{-2}(t)+y^{\delta_{1}}(t)-\frac{1}{1000} \frac{1}{t^{\frac{1}{2}}(1-t)^{\frac{1}{2}}}\right)=0, \quad 0<t<1, \\
& y(0)=\int_{0}^{1} y^{\frac{1}{3}}(s) d A(s), \\
& y(1)=\int_{0}^{1}(y(s))^{\frac{1}{2}} d B(s), \quad d A(s)=\frac{1}{2} d s, d B(s)=\frac{1}{2} d \sin s,
\end{aligned}
$$

where $\delta_{1}>1$.

Let $q(t)=\frac{7}{64}, F(t, y)=y^{-2}+y^{\delta_{1}}, g(y)=y^{-2}, h(y)=y^{\delta_{1}}, c_{0}=\max \left\{\int_{0}^{1} d A(s), \int_{0}^{1} d B(s)\right\}=\frac{1}{2}$, $b_{0}=\frac{7}{384}, \gamma(t)=\frac{1}{1000} \frac{1}{t^{\frac{1}{2}}(1-t)^{\frac{1}{2}}}, c_{1}=\int_{0}^{1} q(s) \gamma(s) d s \leq \frac{1}{500}$. It is easy to see that $\left(\mathrm{C}_{1}\right),\left(\mathrm{C}_{3}\right)-\left(\mathrm{C}_{4}\right)$ 
and (4.10) hold, and since $F(t, y) \geq \frac{1}{\left(4 c_{1}\right)^{2}} \geq(125)^{2}$ for $(t, y) \in[0,1] \times\left(0,4 c_{1}\right]$ and

$$
\max _{t \in[0,1]} \int_{0}^{1} k(t, s) q(s)(125)^{2} d s=\frac{7}{64} 125^{2} \max _{t \in[0,1]} \int_{0}^{1} k(t, s) d s>2 c_{1},
$$

we find that $\left(\mathrm{C}_{2}\right)^{\prime}$ is true.

Since

$$
\frac{1}{1+\frac{h(1)}{g(1)}} \int_{c_{0} \max \left\{1^{\frac{1}{3}}, 1^{\frac{1}{2}}\right\}}^{1} \frac{1}{g\left(\frac{1}{2} y\right)} d y=\frac{1-\frac{1}{8}}{24}=\frac{7}{192}
$$

we have

$$
\sup _{r \in\left(2 c_{1},+\infty\right)} \frac{1}{1+\frac{h(r)}{g(r)}} \int_{c_{0} \max \left\{r^{\frac{1}{3}}, 1^{\frac{1}{2}}\right.}^{r} \frac{1}{g\left(\frac{1}{2} y\right)} d y>b_{0},
$$

which guarantees that (4.11) is true. Moreover, since

$$
\lim _{y \rightarrow+\infty} \frac{F(t, y)}{y}=+\infty
$$

uniformly on $[0,1]$, all the conditions of Theorem 4.2 hold. Then (4.21)-(4.22) has at least two positive solutions.

Example 4.2 Consider

$$
\begin{aligned}
& y^{\prime \prime}(t)+\frac{7}{64}\left(y^{-2}(t)+y^{\delta_{1}}(t)-\frac{1}{1000} \frac{1}{t^{\frac{1}{2}}(1-t)^{\frac{1}{2}}}\right)=0, \quad 0<t<1, \\
& y(0)=\int_{0}^{1} y^{3}(s) d A(s), \\
& y(1)=\int_{0}^{1}(y(s))^{\frac{1}{2}} d B(s), \quad d A(s)=\frac{1}{2} d s, d B(s)=\frac{1}{2} d \sin s,
\end{aligned}
$$

where $0<\delta_{1} \leq 1$.

Since $a=3>1$, using Theorem 4.3, we see that (4.23)-(4.24) has at least two positive solutions.

\section{Uniqueness of positive solutions for the singular boundary value problem}

In this section, we consider the uniqueness of positive solution for BVP (1.1)-(1.2).

Lemma 5.1 (see [20]) Suppose that $E$ is a Banach space with a normal and solid cone $P \subseteq E$ and $A: \stackrel{\circ}{P} \times \stackrel{\circ}{P} \rightarrow \stackrel{\circ}{P}$ is a mixed monotone operator. Moreover, suppose there is a constant $\theta$ with $0 \leq \theta<1$ such that

$$
A\left(t x, \frac{1}{t} y\right) \geq t^{\theta} A(x, y), \quad \forall x, y \in \stackrel{\circ}{P}, 0<t<1 .
$$

Then $A$ has a unique fixed point in $\stackrel{\circ}{P}$. 
Theorem 5.1 Suppose that $\left(C_{1}\right),\left(C_{3}\right),\left(C_{4}\right),\left(C_{5}\right)$ hold and $1>\max \{a, b\}>0$. Then $B V P(1.1)-(1.2)$ has a unique positive solution.

Proof It is easy to see that $P$ defined by (2.1) is a normal and solid cone. For $x, y \in \stackrel{\circ}{P}$, define

$$
A(x, y)(t)=(1-t) \alpha[x]+t \beta[y]+\int_{0}^{1} k(t, s) q(s) g(s, x(s), y(s)) d s, \quad t \in[0,1],
$$

where $g$ is given in $\left(C_{5}\right)$. Since $x, y \in \stackrel{\circ}{P}$, we have $\min _{t \in[0,1]} x(t)>0$ and $\min _{t \in[0,1]} y(t)>0$. Then $\left(C_{1}\right)$ guarantees that

$$
\int_{0}^{1} x^{a}(s) d A(s)>0, \quad \int_{0}^{1} y^{b}(s) d B(s)>0,
$$

which implies that

$$
\min _{t \in[0,1]}[(1-t) \alpha[x]+t \beta[y]]>0 .
$$

Therefore, $A(x, y) \in \stackrel{\circ}{P}$.

Let $\theta_{1}=\max \{\theta, \max \{a, b\}\}$. For $0<\lambda<1$ and $x \in \stackrel{\circ}{P}, y \in \stackrel{\circ}{P}$, from $\left(C_{5}\right)$, we have

$$
\begin{aligned}
A\left(\lambda x, \frac{1}{\lambda} y\right)(t) & =(1-t) \alpha[\lambda x]+t \beta[\lambda y]+\int_{0}^{1} k(t, s) g\left(s, \lambda x(s), \frac{1}{\lambda} y(s)\right) d s \\
& \geq(1-t) \lambda^{a} \alpha[x]+t \lambda^{b} \beta[y]+\lambda^{\theta} \int_{0}^{1} k(t, s) g(s, x(s), y(s)) d s \\
& \geq(1-t) \lambda^{\theta_{1}} \alpha[x]+t \lambda^{\theta_{1}} \beta[y]+\lambda^{\theta_{1}} \int_{0}^{1} k(t, s) g(s, x(s), y(s)) d s \\
& =\lambda^{\theta_{1}} A(x, y)(t), \quad \forall t \in[0,1] .
\end{aligned}
$$

From Lemma 5.1, $A$ has a unique fixed point $x^{*}$ in $\stackrel{\circ}{P}$, which satisfies

$$
\begin{aligned}
x^{*}(t) & =(1-t) \alpha\left[x^{*}\right]+t \beta\left[x^{*}\right]+\int_{0}^{1} k(t, s) q(s) g\left(s, x^{*}(s), x^{*}(s)\right) d s \\
& =(1-t) \alpha\left[x^{*}\right]+t \beta\left[x^{*}\right]+\int_{0}^{1} k(t, s) q(s) f\left(s, x^{*}(s)\right) d s, \quad t \in[0,1] .
\end{aligned}
$$

Then $x^{*}(t)$ is the unique positive solution of BVP (1.1)-(1.2). The proof is complete.

Example 5.1 Consider

$$
\begin{aligned}
& y^{\prime \prime}(t)+\frac{7}{64}\left(y^{-\delta_{1}}(t)+y^{\delta_{2}}(t)\right)=0, \quad 0<t<1, \\
& y(0)=\int_{0}^{1} y^{\delta_{3}}(s) d A(s), \\
& y(1)=\int_{0}^{1}(y(s))^{\delta_{4}} d A(s), \quad d A(s)=d \sin s^{2}, d B(s)=d e^{s},
\end{aligned}
$$

where $0<\max \left\{\delta_{1}, \delta_{2}, \delta_{3}, \delta_{4}\right\}<1$. 
It is easy to see that all conditions of Theorem 5.1 hold, which guarantees that (5.1)-(5.2) has a unique positive solution.

\section{Competing interests}

The authors declare that they have no competing interests.

\section{Authors' contributions}

All authors contributed equally to the writing of this paper. All authors read and approved the final manuscript.

\section{Author details}

${ }^{1}$ Department of Mathematics, Shandong Normal University, Jinan, 250014, P.R. China. ${ }^{2}$ School of Mathematics, Statistics and Applied Mathematics, National University of Ireland, Galway, Ireland. ${ }^{3}$ Department of Mathematics, Nonlinear Analysis and Applied Mathematics (NAAM), King Abdulaziz University, Jeddah, Saudi Arabia. ${ }^{4}$ Department of Mathematics, Texas A\&M University-Kingsville, Kingsville, TX 78363, USA

Received: 9 March 2014 Accepted: 29 May 2014 Published online: 25 September 2014

\section{References}

1. Yang, Z: Existence and uniqueness of positive solutions for an integral boundary value problem. Nonlinear Anal., Theory Methods Appl. 69(11), 3910-3918 (2006)

2. Goodrich, CS: On a nonlocal BVP with nonlinear boundary conditions. Results Math. 63, 1351-1364 (2013)

3. Infante, G: Nonlocal boundary value problems with two nonlinear boundary conditions. Commun. Appl. Anal. 12 279-288 (2008)

4. Kong, L: Second order singular boundary value problems with integral boundary conditions. Nonlinear Anal., Theory Methods Appl. 72(5), 2628-2638 (2010)

5. Webb, JRL: Positive solutions of a boundary value problem with integral boundary conditions. Electron. J. Differ. Equ. 2011, 1-10 (2011)

6. Agarwal, RP, O'Regan, D: Existence theory for single and multiple solutions to singular positone boundary value problems. J. Differ. Equ. 175, 393-414 (2001)

7. Jiang, J, Liu, L, Wu, Y: Positive solutions for second-order singular semipositone differential equations involving Stieltjes integral conditions. Abstr. Appl. Anal. 2012, Article ID 696283 (2012)

8. O'Regan, D: Existence Theory for Nonlinear Ordinary Differential Equations. Kluwer Academic, Dordrecht (1997)

9. Liu, L, Hao, X, Wu, Y: Positive solutions for singular second order differential equations with integral boundary conditions. Math. Comput. Model. 57, 836-847 (2013)

10. Taliaferro, S: A nonlinear singular boundary value problem. Nonlinear Anal. 3, 897-904 (1979)

11. Webb, JRL, Infante, G: Positive solutions of nonlocal boundary value problems: a unified approach. J. Lond. Math. Soc 74, 673-693 (2006)

12. Webb, JRL, Infante, G: Positive solutions of nonlocal boundary value problems involving integral conditions. Nonlinear Differ. Equ. Appl. 15, 45-67 (2008)

13. Baxley, JV: A singular nonlinear boundary value problem: membrane response of a spherical cap. SIAM J. Appl. Math. 48, 497-505 (1988)

14. Bobisud, LE, Calvert, JE, Royalty, WD: Some existence results for singular boundary value problems. Differ. Integral Equ. 6, 553-571 (1993)

15. Boucherif, A: Second-order boundary value problems with integral boundary conditions. Nonlinear Anal., Theory Methods Appl. 70, 364-371 (2009)

16. Ding, Y: Positive solutions for integral boundary value problem with $\varphi$-Laplacian operator. Bound. Value Probl. 2011, Article ID 827510 (1988)

17. Guo, D, Lakshmikantham, V: Nonlinear Problems in Abstract Cones. Academic Press, Boston (1988)

18. Agarwal, RP, O'Regan, D: A survey of recent results for initial and boundary value problems singular in the dependent variable. In: Original Research Article Handbook of Differential Equations: Ordinary Differential Equations, vol. 1 pp. 1-68 (2000)

19. Deimling, K: Nonlinear Functional Analysis. Springer, New York (1985)

20. Guo, D: Fixed point of mixed monotone operators and applications. Appl. Anal. 31, 215-224 (1988)

doi:10.1186/s13661-014-0148-9

Cite this article as: Yan et al.: Multiplicity and uniqueness results for the singular nonlocal boundary value problem involving nonlinear integral conditions. Boundary Value Problems 2014 2014:148. 